\title{
Influence of Familial Risk for Depression on Cortico-Limbic Connectivity During Implicit Emotional Processing
}

\author{
Carolin Wackerhagen*,I, Torsten Wüstenberg', Sebastian Mohnke', Susanne Erk', Ilya M Veer', \\ Johann D Kruschwitz', Maria Garbusow', Lydia Romund', Kristina Otto², Janina I Schweiger², Heike Tost', \\ Andreas Heinz ${ }^{1,3}$, Andreas Meyer-Lindenberg ${ }^{2,3}$, Henrik Walter ${ }^{1,3}$ and Nina Romanczuk-Seiferth ${ }^{1,3}$ \\ 'Division of Mind and Brain Research, Department of Psychiatry and Psychotherapy, Charité- Universitätsmedizin Berlin, Campus Mitte, Berlin, \\ Germany; ${ }^{2}$ Department of Psychiatry and Psychotherapy, Central Institute of Mental Health, Mannheim, Germany
}

\begin{abstract}
Imbalances in cortico-limbic activity and functional connectivity (FC) supposedly underlie biased emotional processing and present putative intermediate phenotypes (IPs) for major depressive disorder (MDD). To prove the validity of these IPs, we assessed them in familial risk. In 70 healthy first-degree relatives of MDD patients and 70 controls, brain activity and seed-based amygdala FC were assessed during an implicit emotional processing task for $\mathrm{fMRI}$ containing angry and fearful faces. Using the generalized psychophysiological interaction approach, amygdala FC was assessed (a) across conditions to provide comparable data to previous studies and (b) compared between conditions to elucidate its implications for emotional processing. Associations of amygdala FC with self-reported negative affect were explored post hoc. Groups did not differ in brain activation. In relatives, amygdala FC across conditions was decreased with superior and medial frontal gyrus (SFG, MFG) and increased with subgenual and perigenual anterior cingulate cortex (sgACC, pgACC). NA was inversely correlated with amygdala FC with MFG, pgACC and their interaction in relatives. Relatives showed aberrant condition-dependent modulations of amygdala FC with visual cortex, thalamus and orbitofrontal cortex. Our results do not support imbalanced cortico-limbic activity as IP for MDD. Diminished amygdala-dorsomedial prefrontal FC in relatives might indicate insufficient regulatory capacity, which appears to be compensated by ventromedial prefrontal regions. Differential task-dependent modulations of amygdala FC are discussed as a stronger involvement of automatic instead of voluntary emotional processing pathways. Reliability and etiological implications of these results should be investigated in future studies including longitudinal designs and patient-risk-control comparisons.

Neuropsychopharmacology (2017) 42, 1729-1738; doi:I0.1038/npp.2017.59; published online 5 April 2017
\end{abstract}

\section{INTRODUCTION}

According to the cortico-limbic dysregulation model of major depressive disorder (MDD), negative emotional salience induces limbic and ventromedial prefrontal hyperactivity to override dorsal cortical control, which intensifies negative emotional processing and facilitates negative affect (Disner et al, 2011; Mayberg, 1997). While this model has meta-analytic support in MDD patients (Graham et al, 2013), its role in the pathogenesis of depression is still to be explored. Here, we investigated whether familial risk for depression is associated with cortico-limbic dysregulation.

Although the heritability of depression is relatively low (Sullivan et al, 2012) and no single nucleotide polymorphism has yet been significantly associated with MDD in genomewide studies (Levinson et al, 2014), there is robust evidence

\footnotetext{
* Correspondence: C Wackerhagen, Division of Mind and Brain Research, Department of Psychiatry and Psychotherapy, CharitéUniversitätsmedizin Berlin, Campus Mitte, Charitéplatz 1, Berlin 101 17 , Deutschland, Germany, Tel: +49 30450517 229, Fax: +49 30450517 906, E-mail: carolin.wackerhagen@charite.de

${ }^{3}$ These authors contributed equally to this work.

Received 29 November 2016; revised 20 February 2017; accepted 7 March 2017; accepted article preview online 15 March 2017
}

of increased familial load (Sullivan et al, 2000). First-degree kinship with an MDD patient is one of the strongest predictors of early onset (Klein et al, 2013) and multiplies risk to develop the disorder by the factor 2 to 3 in offspring (Wilde et al, 2014), siblings (Li et al, 2008), and first-degree relatives in general (Sullivan et al, 2000). It has been shown that, similar to patients with MDD or anxiety disorders (Mathews and MacLeod, 2005), individuals at familial risk for depression exhibit a negative bias when processing emotional information (Foland-Ross et al, 2012). In line with the cortico-limbic dysregulation model, functional imaging studies of emotional conflict and regulation in first-degree relatives suggest that hyperactivity in bottom-up emotionprocessing structures such as amygdala, insula and ventral prefrontal cortex (Joormann et al, 2012; Monk et al, 2008; Pilhatsch et al, 2014), or a hypo-activity of executive control structures in the dorsal PFC (Amico et al, 2012; Joormann et al, 2012; Mannie et al, 2008, 2011) may contribute to this bias. However, these studies were methodologically variable with inconsistent results, relatively small sample sizes (risk group ranged from 11 to 30 subjects), and focused on child and adolescent offspring of MDD patients. Furthermore, although the cortico-limbic dysregulation model is widely supported by functional connectivity (FC) studies in MDD 
patients (Kaiser et al, 2015), to our knowledge, no study has yet assessed FC during emotional processing in first-degree relatives.

A related field of etiological research is imaging genetics, an approach aiming to identify intermediate phenotypes (IP) of genetic risk for psychiatric disorders. IPs are conceptualized as genetically driven biological traits more directly associated with genetic risk variants than clinical categories, thereby 'bridging the gap' between genotype and clinical phenotype (Meyer-Lindenberg and Weinberger, 2006). Firstdegree relatives, who share about $50 \%$ of genetic variance with their affected family member, provide crucial evidence for the validation of IPs (Gottesman and Gould, 2003). A pioneering imaging genetics finding, the association of the serotonin transporter polymorphism 5-HTTLPR with amygdala hyperactivity in response to fearful and angry faces (Hariri et al, 2002), has not yet been validated in first-degree relatives.

Aberrant amygdala-prefrontal FC has been proposed as connectomic IP for depression (Fornito and Bullmore, 2012), although locations and directions of reported effects have been heterogeneous. In 5-HTTLPR risk allele carriers, reduced amygdala FC with subgenual anterior cingulate cortex (sgACC) has been interpreted as reduced regulatory control (Pezawas et al, 2005). On the other hand, increased amygdala FC with the ventromedial PFC has been interpreted as compensatory regulation (Friedel et al, 2009; Heinz et al, 2005; Schardt et al, 2010), but would also fit the hypothesis of increased salience signaling between limbic and ventral prefrontal bottom-up processing structures (Disner et al, 2011). Since FC is a correlative measure, hypotheses regarding its corresponding neurobiological processes need to be confirmed by complementary approaches.

Here, we assessed brain activity and amygdala FC during an implicit emotional processing paradigm, during which subjects matched angry and fearful faces (emotion condition) or geometric shapes (control condition; Hariri et al, 2002). To provide more detailed information about the functional integration of the amygdala, we did not only assess amygdala FC across task conditions (faces matching, shapes matching), as was done in previous studies, but also measured its taskdependent changes between conditions (faces matching $>$ shapes matching) using the generalized psychophysiological interaction (gPPI) approach (McLaren et al, 2012).

Seventy healthy first-degree relatives of MDD patients and 70 controls were assessed. We hypothesized that: (1) relatives show amygdala hyperactivity and prefrontal hypoactivity and (2) relatives show aberrant amygdala-PFC FC across task conditions. (3) We further explored group-by-condition interactions of task-dependent amygdala FC. Together, this would provide support for cortico-limbic dysregulation being a familial risk biomarker and IP for depression. In addition, we performed post hoc exploratory analyses of (1) associations of FC measures with self-reported negative affect, (2) differences in amygdala FC between offspring and siblings of MDD patients, and (3) associations between amygdala response and amygdala FC.

\section{MATERIALS AND METHODS}

\section{Participants}

The sample consisted of 70 unaffected adult first-degree relatives of patients with MDD (45 females, mean age: 28.0 years) and 70 control participants (41 females, mean age $=29.7$ years) without a personal or familial history of psychiatric disorders. It was selected from a multicenter study of neurogenetic causes of psychiatric disorders conducted in Berlin, Bonn, and Mannheim, Germany (Erk et al, 2014). All subjects were native German speakers; for the purpose of genetic homogeneity only subjects with German nationality and German/European ancestry up to the second generation were included. We excluded subjects with clinically relevant depression scores (Beck Depression Inventory (BDI-I) (Hautzinger et al, 1994) > 18), incidental neuroradiological findings, and missing or qualitatively poor data (see supplementary Figure S1). In order to control for potentially confounding factors such as study site, age, gender, level of education, and depressive symptoms, a sample of 70 controls was selected from a larger pool of control subjects based on matching to relatives with respect to these variables. Subjects were included if index patients had a diagnosis of MDD, and no diagnosis of bipolar disorder, schizophrenia, or substance dependence (except for tobacco), which was confirmed using the German version of the Structured Clinical Interview for DSM-IV-TR Axis I Disorders (SCID-I, (Wittchen et al, 1997)), conducted by experienced clinicians, or, in case index patients were unavailable, by medical reports. Except for familial history of MDD in relatives, both groups had no personal or familial histories of lifetime axis I disorders as confirmed by the SCID-I screening. Participants gave written informed consent before study inclusion. The study was approved by the local ethics committees of the participating centers.

\section{Measures of Negative Affect}

As state measures of negative affect the BDI-I (Hautzinger et al, 1994), the depression scale of the Symptom Checklist90 (SCL-90-R, Derogatis, 1977), and state anxiety (STAI-S, Spielberger et al, 1970) were assessed directly before scanning. As trait measures of negative affect, NEO-FFI neuroticism (Costa and McCrae, 1992), and trait anxiety (STAI-T, Spielberger et al, 1970) were assessed during a neuropsychological assessment session within 1-2 weeks after MRI scanning. The social readjustment rating scale (SRRS, Holmes and Rahe, 1967) was assessed for the past 24 months as a measure of stressful life events.

\section{Emotional Processing Task}

FMRI data were taken from a larger protocol, including 6 tasks and a resting state session (total duration $1 \mathrm{~h}$ ). The implicit emotional processing task was an adaptation of the face matching task (Hariri et al, 2000), in which participants were instructed to identify matching pairs in trios of stimuli. In the emotion condition, trios contained gray-scale photographs of angry or fearful faces, counterbalanced for gender and emotional valence. In the control condition, trios contained geometric shapes (circles, horizontal and vertical ellipses). Four blocks per condition were presented in 
alternation. Each block consisted of one instruction $(2 \mathrm{~s})$ and 6 trials ( $5 \mathrm{~s}$ each). The whole experiment lasted $274 \mathrm{~s}$ (see Supplementary Material for details).

\section{MRI Parameters}

Functional and structural MRI was performed at 3T Siemens Trio scanners (Erlangen, Germany) using identical scanning protocols. During the task, 135 whole-head gradient echo planar imaging (EPI) volumes were acquired (28 slices, matrix size $=64 \times 64$, slice thickness $=3 \mathrm{~mm}$, interslice gap $=1 \mathrm{~mm}$, field of view $=192 \mathrm{~mm}$, voxel size $=3 \times 3 \times$ $3 \mathrm{~mm}^{3}, \mathrm{TR}=2 \mathrm{~s}, \mathrm{TE}=30 \mathrm{~ms}$, flip angle $=80^{\circ}$ ). In addition, a field inhomogeneity map was acquired, as well as a T1-weighted anatomical 3D image (for coregistration and normalization purposes), using a magnetization prepared rapid acquisition gradient echo (MP-RAGE) sequence with an isotropic spatial resolution of $1 \mathrm{~mm}^{3}$ (field of view $=192$, $\mathrm{TR}=1.57 \mathrm{~s}, \mathrm{TE}=2.74 \mathrm{~ms}$, flip angle $=15^{\circ}$ ).

\section{Image Processing}

Processing of brain images was conducted using statistical parametric mapping methods as implemented in SPM12b (http://www.fil.ion.ucl.ac.uk/spm/software/spm12/). Images underwent correction for acquisition delay, correction for head motion, unwarping using the field inhomogeneity map, coregistration of the EPI to the individual T1 image, normalization into standard space $\left(3 \times 3 \times 3 \mathrm{~mm}^{3}\right.$ voxels $)$, and spatial smoothing ( $8 \mathrm{~mm}$ FWHM).

\section{Analyses of Task-Related Brain Activity}

Brain responses were estimated within the Generalized Linear Modeling (GLM) framework implemented in SPM (www.fil.ion.ucl.ac.uk/spm/). The single subject model consisted of regressors for the emotion condition, the control condition, instructions, button presses, and six head motion parameters (Supplementary Figure S2). Before fitting the model, voxel time series were high-pass filtered (cutoff $128 \mathrm{~s}$ ) and temporal dependencies were removed using a first order autoregressive model. Model parameters were estimated using a restricted maximum likelihood algorithm. Linear contrast images were computed for each task condition ('faces'; 'shapes') and entered into group analysis.

\section{Analyses of Task-Related Functional Connectivity}

For each subject amygdala FC was assessed both across and between conditions using a generalized psycho-physiological interaction (gPPI) framework (http://www.nitrc.org/pro jects/gppi/; McLaren et al, 2012; see supplements for details). As physiological term, time-series were extracted from the right and left amygdala (see ROI description) at location of maximum task effect (faces $>$ shapes), which was predominantly (97\%) in the basolateral amygdala (see Supplementary Material). As psychological terms, all regressors modeling the task were included. As PPI terms, the psychological term was convolved with the physiological term (right or left amygdala time-series, respectively) and the canonical hemodynamic response function implemented in SPM. Head motion parameters were included as regressors of no interest. Linear contrast images were computed for each amygdala separately: (1) the effect across conditions ('PPI faces \& PPI shapes'), and (2) the differential effect between conditions ('PPI faces $>$ PPI shapes'). The calculated contrast images were subsequently entered into group analyses.

\section{Group Analyses}

In order to use equivalent statistical methods for both modalities, we performed analyses of variance for repeated measures (rmANOVAs) for all group level analyses. For brain activation, one rmANOVA with the between-subject factor, 'group' (relatives/controls) and the within-subject factor 'condition' (faces/shapes) was conducted. The linear contrast images 'activity faces' and 'activity shapes' were used as dependent variables in this analysis. For FC, two rmANOVAs were conducted to assess the FC effects across and between conditions. Both analyses included the betweensubject factor 'group' and the within-subject factor 'seed location'. For the across conditions analysis, linear contrast images 'PPI faces \& PPI shapes' were used as dependent variables. For FC effects between conditions, the linear contrast 'PPI faces $>$ PPI shapes' were used instead. To eliminate task-unspecific between-subject variance, in all rmANOVAs the individual mean over conditions or seed locations were modeled additionally.

\section{Regions of Interest and Statistical Thresholds}

For PPI seed regions and hypothesis-driven group analyses, literature-based probabilistic ROIs of the right and left amygdala were used, which were composed of coordinates reported in studies of the faces matching task (in order to ensure that they corresponded to regions relevant for emotional faces processing), as well as restricted by anatomical boundaries. A ROI of the PFC for hypothesesdriven group analyses was created using the automatic anatomical labeling atlas as implemented in the WFU pickatlas for SPM (see Supplementary Material).

To decrease the alpha error probability, we applied a Bonferroni-correction for six tests (two comparisons, respectively, for activity, FC across, and FC between conditions, $\quad p<0.0083)$ to voxel-wise whole-brain FWE-corrected results.

\section{Post hoc Exploratory Analyses}

We performed exploratory analyses of brain-behavior associations, differences in amygdala FC between offspring and siblings of MDD patients, and potential associations between amygdala response and amygdala FC. For brain-behavior associations, first, a principal component analysis with BDI, SCL-Depression, STAI-T and Neuroticism was performed to provide a comprehensive measure of negative affect (NA). Then, amygdala functional measures were entered as predictors into a stepwise Generalized Linear Model with NA as dependent variable for relatives and controls separately, which resulted in two models, which were compared to a constant model via F-Test (alpha error probabilities were Bonferroni-corrected for multiple comparisons $\left(p_{\text {corr }}=0.025\right)$ ) and were compared via Likelihood-ratio-test. Differences between offspring and siblings were assessed via two-sample 
t-tests. Associations between amygdala response and amygdala FC were assessed via Pearson correlations. All exploratory analyses are described in detail in Supplementary Material.

\section{RESULTS}

\section{Sample Characteristics}

As shown in Table 1, groups did not differ with respect to age, gender, study site, or years of education. All negative affect scores were below clinical thresholds and did not significantly differ between groups. Groups did not differ in stressful life events (SRRS scores) and task performance.

\section{Task-Related Brain Activity}

Across groups, in the face $>$ shapes contrast, increased activation was found in the visual cortex (cuneus, fusiform, occipital, lingual, and calcarine gyrus), subcortical structures (thalamus, hippocampus, and amygdala), pre- and postcentral regions, the dorsal prefrontal cortex (PFC; BA6, BA8, BA9, BA46), and temporal areas (see Supplementary Table S3 for coordinates and statistics). Brain activity in this contrast did not differ between groups, neither in whole-brain, nor in ROI analyses of the amygdala and the PFC at $p_{\mathrm{FWE}}<0.05$.

\section{Task-Related Amygdala Functional Connectivity}

In the faces compared to the shapes condition, across groups, bilateral amygdala FC was increased with the primary visual cortex (V1), inferior and middle occipital gyrus, and fusiform gyrus, while it was decreased with midline structures (precuneus, cuneus, posterior and mid cingulate gyrus, perigenual anterior cingulate cortex (pgACC; BA32, BA24)), orbitofrontal cortex (OFC; BA10, BA11), and temporal regions (Figure 1, Supplementary Table S4 for coordinates and statistics).

In group comparisons of amygdala FC across conditions, relatives showed decreases in dorsal and increases in ventral parts of the PFC. Decreases were observed in the precentral gyrus (BA6), superior frontal gyrus (BA10), and medial frontal gyrus (MFG; Figure 2, Table 2). Increases were observed in the sgACC (BA24, BA25) and pgACC (BA32, $\mathrm{BA} 24)$, the right temporal pole (BA38), and clusters at the occipital-parietal junction, including angular gyrus (BA39). Although amygdala-pgACC FC was increased in relatives across conditions, task-dependent modulations (decrease during faces processing) did not differ between groups.

Group-by-condition interactions (group comparisons of PPI faces $>$ PPI shapes) are depicted in Figure 3. In the left superior frontal gyrus (SFG), amygdala FC was positive during shapes matching and decreased during faces matching in controls, while it did not significantly differ between conditions in relatives. In the thalamus and visual cortex, controls showed stronger amygdala FC during faces matching compared to shapes matching, while this pattern was inversed in relatives. Amygdala FC with the OFC did not differ between conditions in controls, while it decreased during faces matching in relatives.

All group effects in amygdala FC were significant at $p<0.006$ (voxel-wise corrected across the whole brain) and thus survived additional Bonferroni-correction for the number of six comparisons $(p<0.0083)$.
Table I Demographic and Psychological Sample Characteristics

\begin{tabular}{|c|c|c|c|c|c|}
\hline Characteristic & $\begin{array}{l}\text { Controls } \\
(N=70)\end{array}$ & $\begin{array}{c}\text { Relatives } \\
(N=70)\end{array}$ & df & $\mathbf{F} \chi^{2}$ & $p$-value \\
\hline \multicolumn{6}{|l|}{ Demographics } \\
\hline \multicolumn{6}{|c|}{$\begin{array}{l}\text { Type of family relationship } \\
\text { to index patient }{ }^{\mathrm{a}} N(\%)\end{array}$} \\
\hline Offspring & & $50(74)$ & & & \\
\hline Sibling & & $17(25)$ & & & \\
\hline Parent & & I (I) & & & \\
\hline Age mean $\pm S D, d f, F$ & $29.70 \pm 8.08$ & $28.03 \pm 8.82$ & 139 & 1.37 & 0.24 \\
\hline $\begin{array}{l}\text { Years of education } \\
\text { mean } \pm S D, d f, F\end{array}$ & $15.28 \pm 2.25$ & $15.49 \pm 2.45$ & 139 & 0.25 & 0.61 \\
\hline \multicolumn{6}{|l|}{$\operatorname{Sex} N(\%), d f, \chi^{2}$} \\
\hline Male & $29(4 I)$ & $25(36)$ & । & 0.48 & 0.49 \\
\hline Female & $41(59)$ & $45(64)$ & & & \\
\hline \multicolumn{6}{|l|}{ Study site, $N(\%), d f, \chi^{2}$} \\
\hline Charité Berlin & $27(38)$ & $27(38)$ & । & 0.42 & 0.81 \\
\hline Zl Mannheim & $18(26)$ & $21(30)$ & & & \\
\hline University of Bonn & $25(36)$ & $22(32)$ & & & \\
\hline
\end{tabular}

State negative affect

$\begin{array}{lrrrrr}\text { BDI } & 2.8 \pm 3.1 & 3.9 \pm 3.4 & 134 & 3.8 & 0.06 \\ \text { SCL90-R depression }^{b} & 45.5 \pm 8.1 & 46.1 \pm 8.4 & 134 & 0.15 & 0.70 \\ \text { STAI-S }^{c} & 31.7 \pm 5.92 & 31.4 \pm 5.6 & 100 & 0.11 & 0.74\end{array}$

Trait negative affect

$\begin{array}{llllll}\text { STAI-T } & 33.7 \pm 9.25 & 35.55 \pm 8.52 & 134 & 1.5 & 0.22\end{array}$

$\begin{array}{lllllll}\text { NEO Neuroticism } & 14.7 \pm 7.2 & 16.9 \pm 7.6 & 134 & 2.9 & 0.10\end{array}$

Task performance mean $\pm S D, d f, F$

Reaction time (s)

$\begin{array}{llllll}\text { Faces matching } & 1.25 \pm .22 & 1.22 \pm .28 & 139 & 0.47 & 0.50\end{array}$

$\begin{array}{lllllll}\text { Shapes matching } & 1.10 \pm .20 & 1.11 \pm .26 & 139 & 0.01 & 0.93\end{array}$
Percentage of correct responses

$\begin{array}{llrlll}\text { Faces matching } & 98.87 \pm 2.4 & 98.99 \pm 2.20 & 139 & 0.10 & 0.76 \\ \text { Shapes matching } & 97.20 \pm 3.8 & 97.62 \pm 3.7 & 139 & 0.43 & 0.51\end{array}$

Abbreviations: BDI, Beck Depression Inventory; NEO, NEO Five Factory Inventory; SCL90-R Depression, Symptom Checklist 90 Revised Depression Scale; SRRS, Social Readjustment Rating Scale; STAI-S, State Trait Anxiety Inventory — State Anxiety; STAI-T, State Trait Anxiety Inventory_-Trait Anxiety; Zl, Central Institute of Mental Health.

anformation about family relationship missing for 2 participants.

${ }^{b}$ Raw scores were transformed into age- and gender- adjusted T-distributed norm scores.

'STAI-S was not acquired at study site Mannheim. 


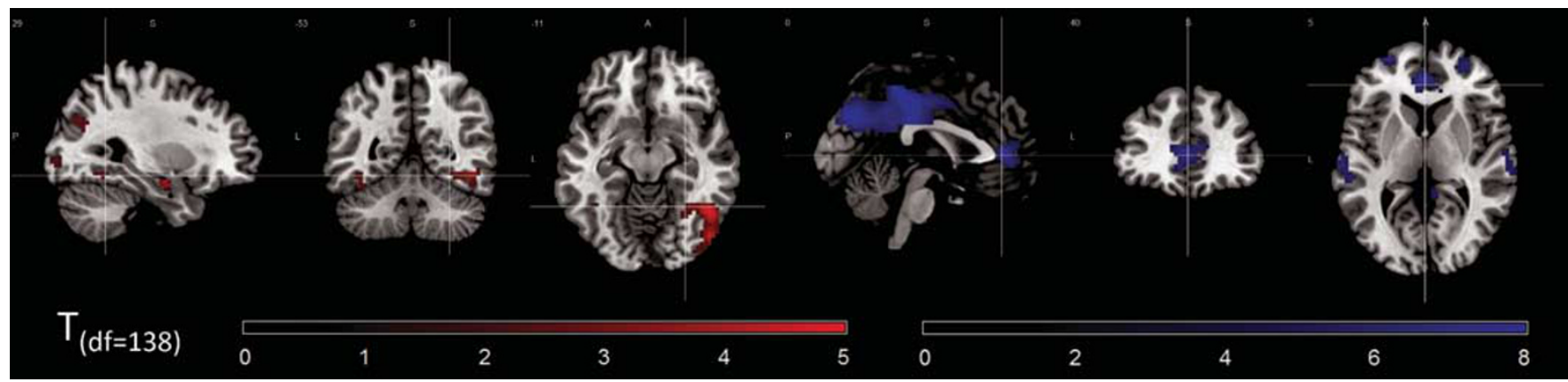

Figure I Main effect of task (across groups) on bilateral amygdala functional connectivity. Increases (red) and decreases (blue) of amygdala functional connectivity in the emotion condition compared to the control condition. MNI coordinates of crosshairs: left panel: $x=29, y=-53, z=-\mid \mathrm{I}$; right panel: $x=0, y=40, z=5$. Significance threshold: $p<0.05$, whole-brain FWE-corrected.

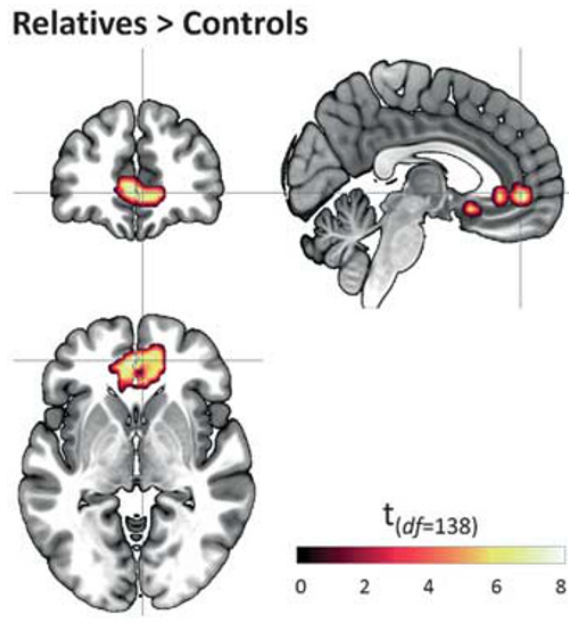

\section{Relatives < Controls}

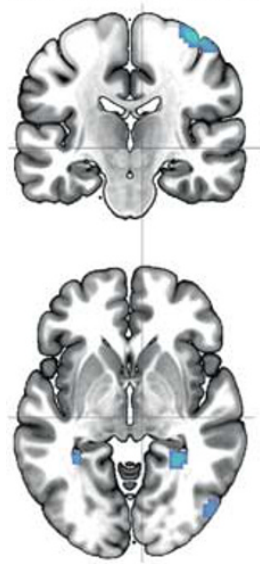

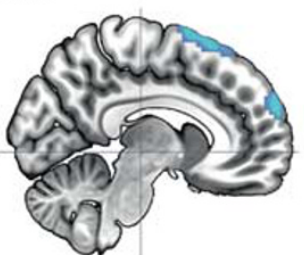

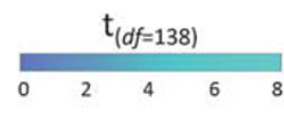

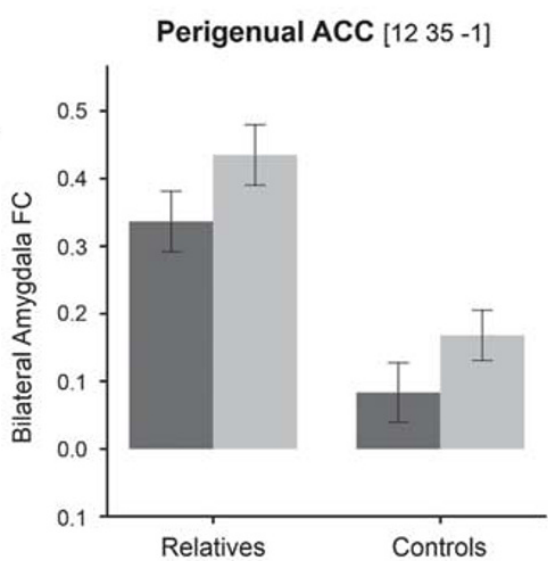

Superior Frontal Gyrus [42 -10 65]

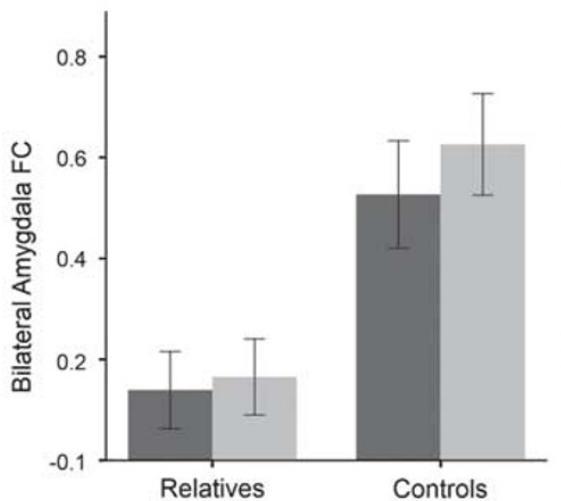

Faces matching $\square$ Shapes matching

Subgenual ACC [3 14 -13]

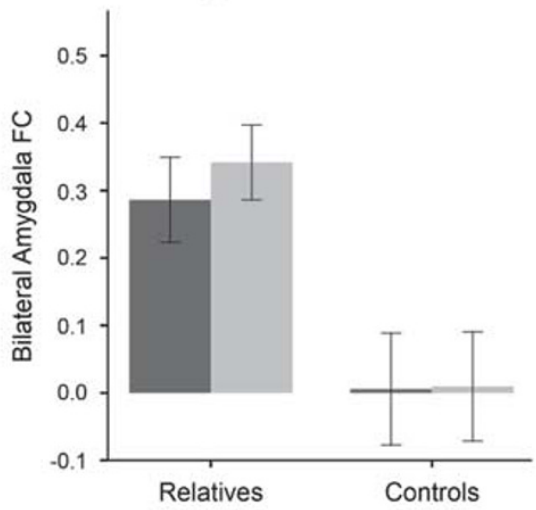

Medial Frontal Gyrus [6 62 26]

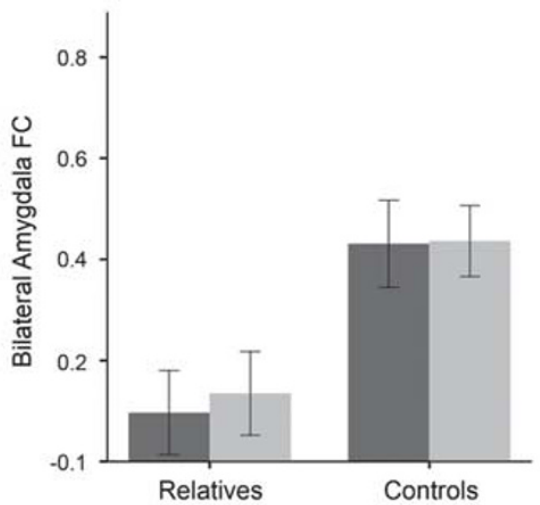

Figure 2 Group differences in amygdala functional connectivity across task conditions. Results are significant at a whole-brain FWE-corrected significance threshold of $p<0.006$. Error bars indicate standard errors of the mean. ACC, anterior cingulate cortex; amygdala FC, amygdala functional connectivity; df, degrees of freedom.

\section{Post hoc Exploratory Results}

Analyses of brain-behavior associations showed that only in relatives variance in negative affect (NA) was significantly explained by a model including the main effects of amygdalapgACC and amygdala-MFG connectivity (across conditions) and their interaction $\left(F_{2,60}=3.71, p=0.016\right)$. This model showed better performance than the final model for controls $\left(\chi^{2}=16.43, p<0.001\right)$. Figure 4 shows a graphical representation of the model.

Amygdala FC at locations of group differences did not differ between offspring and siblings. There were no significant associations between amygdala response and amygdala FC (see Supplementary Material). 
Table 2 Results of Group Analyses of Bilateral Amygdala Functional Connectivity

Group effects of amygdala functional connectivity across conditions

\begin{tabular}{|c|c|c|c|c|c|c|c|c|c|c|c|c|c|c|c|}
\hline \multicolumn{8}{|c|}{ Controls $>$ relatives } & \multicolumn{8}{|c|}{ Relatives $>$ controls } \\
\hline \multirow[t]{2}{*}{ Brain region } & \multirow[t]{2}{*}{$k$} & \multirow[t]{2}{*}{$H$} & \multirow[t]{2}{*}{$T$} & \multirow[t]{2}{*}{$P($ FWE $)$} & \multicolumn{3}{|c|}{ MNI coord. } & \multirow[t]{2}{*}{ Brain region } & \multirow[t]{2}{*}{$k$} & \multirow[t]{2}{*}{$H$} & \multirow[t]{2}{*}{$T$} & \multirow[t]{2}{*}{$P_{\text {(FWE) }}$} & \multicolumn{3}{|c|}{ MNI coord. } \\
\hline & & & & & $x$ & $y$ & $\mathbf{z}$ & & & & & & $x$ & $y$ & $\mathbf{z}$ \\
\hline Precentral Gyr & 217 & $\mathrm{R}$ & 7.9 & $<0.001$ & 42 & -10 & 65 & Mid Occ Gyr (BA39) & 79 & $\mathrm{~L}$ & 8.0 & $<0.001$ & -33 & -85 & 41 \\
\hline SFG (BA6) & & $\mathrm{R}$ & 6.1 & $<0.001$ & 21 & -10 & 74 & Mid Orb Gyr & & $\mathrm{R}$ & 7.3 & $<0.001$ & 0 & 47 & -4 \\
\hline Postcentral Gyr & & $\mathrm{R}$ & 5.9 & 0.001 & 51 & -19 & 59 & Mid Orb Gyr (BA32) & & $\mathrm{R}$ & 6.8 & $<0.001$ & 12 & 38 & -4 \\
\hline SMG (BAI0) & 44 & $\mathrm{R}$ & 6.8 & $<0.00$ I & 6 & 62 & 26 & MTP (BA38) & 25 & $\mathrm{R}$ & 7.4 & $<0.001$ & 30 & ।1 & -34 \\
\hline \multirow[t]{2}{*}{ MFG (BAI0) } & & $\mathrm{R}$ & 5.8 & 0.001 & 21 & 59 & 32 & $\operatorname{sgACC}(B A 24,25)$ & 13 & M & 6.9 & $<0.001$ & 0 & 14 & -13 \\
\hline & & & & & & & & sgACC (BA24, 25) & & $L$ & 6.0 & $<0.001$ & -3 & 14 & -16 \\
\hline
\end{tabular}

Group effects of amygdala functional connectivity between conditions

Emotion $>$ control; controls $>$ relatives

\begin{tabular}{|c|c|c|c|c|c|c|c|c|c|c|c|c|c|c|c|}
\hline \multirow[t]{2}{*}{ Brain region } & \multirow[t]{2}{*}{$k$} & \multirow[t]{2}{*}{$H$} & \multirow[t]{2}{*}{$T$} & \multirow[t]{2}{*}{ P(FWE) } & \multicolumn{3}{|c|}{ MNI coord. } & \multirow[t]{2}{*}{ Brain region } & \multirow[t]{2}{*}{$k$} & \multirow[t]{2}{*}{$H$} & \multirow[t]{2}{*}{$T$} & \multirow[t]{2}{*}{ P(FWE) } & \multicolumn{3}{|c|}{ MNI coord. } \\
\hline & & & & & $x$ & $y$ & z & & & & & & $x$ & $y$ & $\mathbf{z}$ \\
\hline Lingual Gyr & 180 & $\mathrm{~L}$ & 7.7 & $<0.001$ & -12 & -79 & -7 & SMG (BA40) & 12 & $\mathrm{R}$ & 6.6 & $<0.001$ & 63 & -46 & 38 \\
\hline Fusiform Gyr & & $\mathrm{L}$ & 6.7 & $<0.001$ & -30 & -73 & -10 & Precentral Gyr (BA4) & 12 & $\mathrm{R}$ & 5.9 & 0.001 & 57 & -10 & 47 \\
\hline Fusiform Gyr & 123 & $\mathrm{R}$ & 7.4 & $<0.001$ & 27 & -76 & -4 & SFG (BAIO) & II & $\mathrm{L}$ & 5.0 & 0.005 & -21 & 59 & 23 \\
\hline $\operatorname{lnf}$ Occ Gyr & & $\mathrm{R}$ & 5.9 & 0.001 & 42 & -67 & -7 & & & & & & & & \\
\hline Lingual Gyr & & $\mathrm{R}$ & 5.6 & 0.002 & 18 & -85 & -7 & & & & & & & & \\
\hline Cuneus & 80 & $\mathrm{R}$ & 7.3 & $<0.001$ & 21 & -94 & 14 & & & & & & & & \\
\hline Mid Occ Gyr & & $\mathrm{R}$ & 6.0 & $<0.001$ & 30 & -97 & 17 & & & & & & & & \\
\hline OFC (BAII) & 41 & $\mathrm{~L}$ & 5.8 & 0.001 & -39 & 35 & -13 & & & & & & & & \\
\hline MOccG & 88 & $\mathrm{~L}$ & 6.5 & $<0.001$ & -21 & -94 & 5 & & & & & & & & \\
\hline Inf Occ Gyr & & $\mathrm{L}$ & 5.9 & $<0.001$ & -24 & -94 & -4 & & & & & & & & \\
\hline Calcarine Gyr & & $\mathrm{L}$ & 5.3 & 0.006 & -12 & -91 & 11 & & & & & & & & \\
\hline Thalamus & I I & $\mathrm{L}$ & 5.4 & 0.005 & -3 & -13 & 5 & & & & & & & & \\
\hline
\end{tabular}



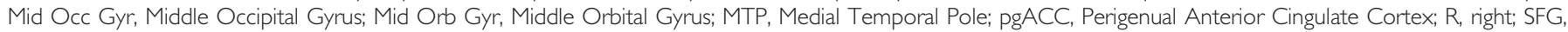
Superior Frontal Gyrus; sgACC, Subgenual Anterior Cingulate Cortex; SMA, Supplementary Motor Area; SMG, Superior Medial Gyrus; OFC, Orbitofrontal Cortex.

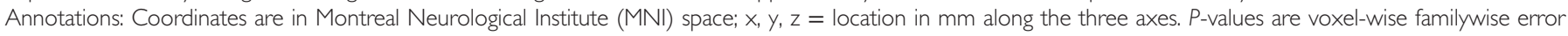
(FWE) corrected across the whole brain.

\section{DISCUSSION}

This study investigated whether cortico-limbic imbalance served as biomarker of familial risk and intermediate phenotype (IP) for depression by assessing cortico-limbic activation and amygdala FC during implicit emotional processing in a sample of MDD relatives and control subjects who were comparable regarding demographics, depression scores, stressful life events and task performance. We found that the task induced activation in distributed brain areas including limbic and prefrontal cortical regions, which did not differ between groups. For the first time, amygdala FC was assessed not only across but also between task conditions to investigate how it relates to the absence and presence of emotional stimuli. During the faces condition, amygdala FC increased with visual regions, while it decreased with cortical midline structures including perigenual anterior cingulate cortex (pgACC). Across conditions, relatives showed decreases in amygdala FC with the dorsomedial PFC (dmPFC; SFG and MFG) and increases with the ventromedial PFC (vmPFC; pgACC, sgACC). Negative affect was inversely correlated with amygdala FC to $\operatorname{pgACC}$, MFG, and their interaction in relatives. Amygdala FC with visual cortex, thalamus, orbitofrontal cortex and superior frontal gyrus showed differential condition-dependent modulations in relatives compared to controls. 


\section{Faces $>$ Shapes Contrast Relatives > Controls}

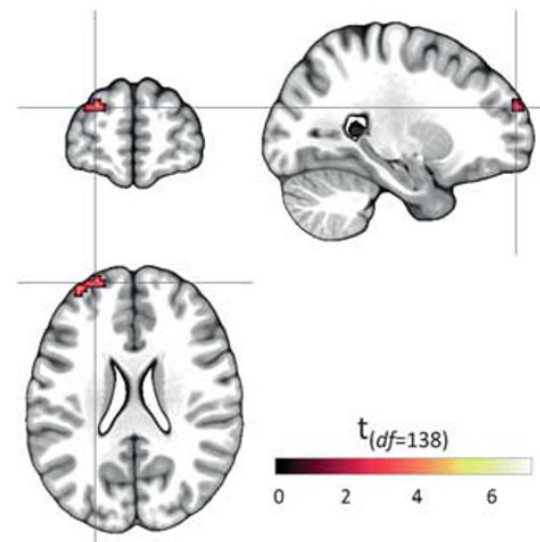

Superior Frontal Gyrus [-21 5923$]$

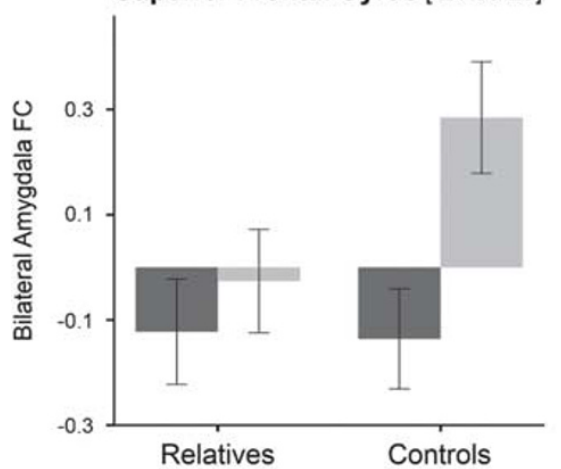

Faces $>$ Shapes Contrast Relatives < Controls



Thalamus $\left[\begin{array}{lll}-3 & -13 & 5\end{array}\right]$

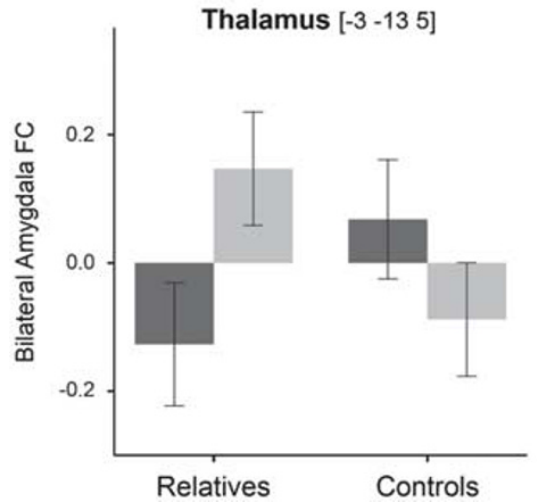

Faces matching

Shapes matching

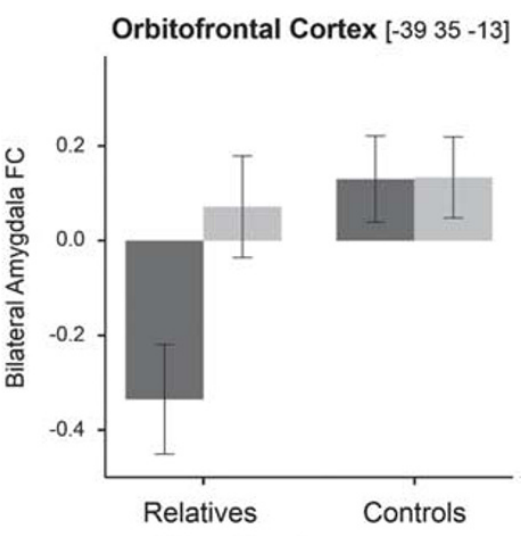

Visual Cortex [-12 -79 -7]

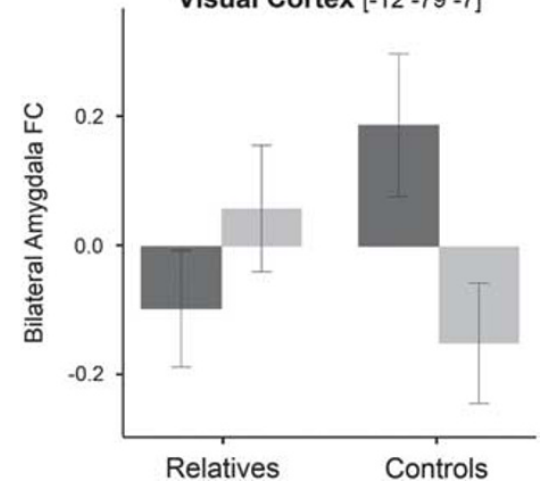

Figure 3 Group by condition interactions in functional amygdala connectivity. Results are significant at a whole-brain FWE-corrected significance threshold of $p<0.006$. Error bars indicate standard errors of the mean. amygdala FC, amygdala functional connectivity; df, degrees of freedom.

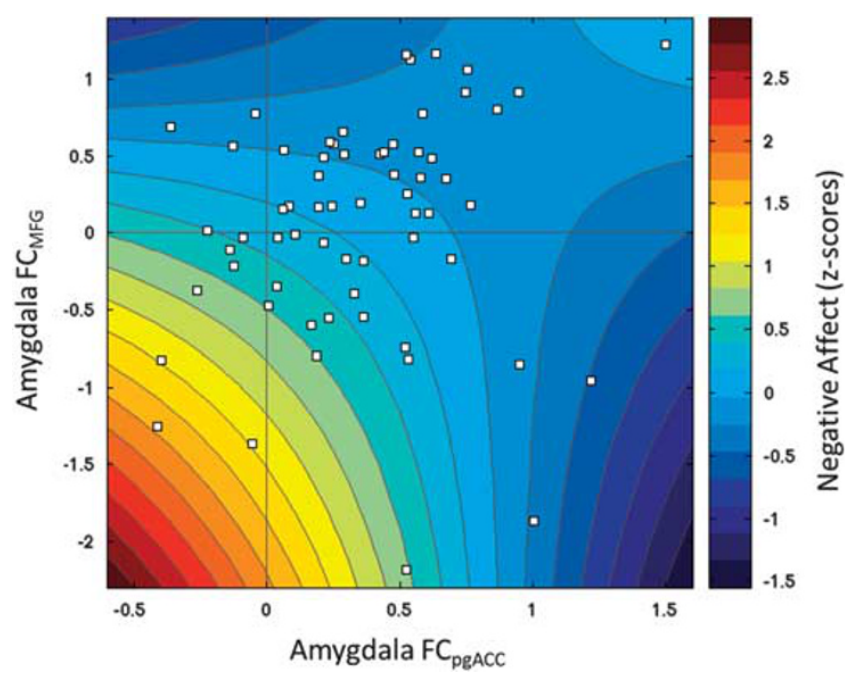

Figure 4 Negative affect as a function of amygdala FC across conditions with pgACC and MFG. Color-coded representation of the final linear model $\left(\mathrm{NA}^{\sim}-0.49 * \mathrm{FC}_{\mathrm{pgACC}}-0.63 * \mathrm{FC}_{\mathrm{MFG}}+0.68 *\left(\mathrm{FC}_{\mathrm{pgACC}} * \mathrm{FC}_{\mathrm{MFG}}\right)+0.30\right.$; $\mathrm{F}_{(1,60)}=3.71 ; p=0.016$ ), and the individual data points (white squares) of relatives. FC, functional connectivity; MFG, medial frontal gyrus; pgACC; perigenual anterior cingulate cortex.

\section{Brain Activation Not Altered in Risk Group}

The main effects of the task were comparable to those reported in a meta-analysis including 105 studies of the face matching task (Fusar-Poli et al, 2009). Since geometric shapes instead of neutral faces were used as control condition, task-induced brain activation may not solely correspond to emotional processing, but to face processing in general. However, activations in amygdala, fusiform gyrus, dorsal and ventral PFC correspond to those shown in a metaanalysis of studies using neutral faces as control (Sabatinelli et al, 2011), so we assume the task evoked emotion-related neural processes.

We did not observe group differences in task-related brain activation-neither whole-brain, nor in ROI-analyses of the amygdalae or PFC $\left(p_{\mathrm{FWE}}<0.05\right)$. Previous studies of brain activation during emotional processing in familial risk were limited to child and adolescent offspring of MDD patients and showed divergent results, likely due to limited sample size and heterogeneity of tasks. Although a few studies did report increased amygdala activation in child and adolescent risk groups during passive viewing of fearful faces (Monk et al, 2008), negative emotional distraction (Pilhatsch et al, 2014), and sad mood induction (Joormann et al, 2012), no amygdala hyperactivity was observed in a number of other emotional processing and automatic attentional control studies (Amico et al, 2012; Lisiecka et al, 2012, 2013; Mannie et al, 2008), including a face matching task comparable to the one used here (Mannie et al, 2011). Our results thus support the conclusion that cortico-limbic activation imbalances do not serve as a familial risk marker for depression. Further, we could not find supporting 
evidence for amygdala hyperactivity as an intermediate phenotype for genetic depression risk, which is in line with a recent meta-analysis showing that the 5-HTTLPR-amygdala association was no longer significant after including unpublished studies (Bastiaansen et al, 2014).

\section{Task-Dependent Changes of Amygdala FC}

To our knowledge, this was the first investigation of condition-dependent changes of amygdala FC during a faces matching task. In the emotion compared to the control condition, amygdala FC was increased with the primary visual cortex (V1) and fusiform gyrus. Given that the amygdala detects salient facial cues automatically, even in masked conditions without explicit knowledge (Whalen et al, 1998), and V1 and fusiform gyrus show face-selective behavior as well (Petro et al, 2013), supposedly under control of the amygdala (Chen et al, 2014), the here observed increased amygdala-visual engagement during faces matching suggests a process of increased salience-induced visual attention.

We further observed that amygdala FC with pgACC, OFC, posterior cingulate, and precuneus decreased during faces compared to shapes matching. A ventral-dorsal segregation has been repeatedly reported in studies of amygdala FC during emotional processing, showing negative FC with dorsal and positive FC with ventral parts of the PFC during emotional processing and social cognition across task conditions (Bzdok et al, 2013; Etkin et al, 2011). Specifically, the dorsal ACC and posterior cingulate have been shown to exert negative effective connectivity over the amygdala during a comparable faces matching task, which was interpreted as inhibitory top-down control (Stein et al, 2007). In 97\% of our sample, the seeds for FC analyses were located in the basolateral amygdala, which is regulated by feedforward inhibition from the medial PFC (McGarry and Carter, 2016). Decreased amygdala-pgACC FC might thus correspond to automatic attentional regulation (Phillips et al, 2008).

\section{Aberrant Amygdala-Prefrontal Coupling in Relatives Across Conditions}

Across conditions, relatives showed reduced FC with clusters in the dorsomedial prefrontal cortex including SFG and MFG and increased amygdala FC with ventromedial prefrontal regions, the sgACC and pgACC. This pattern is consistent with a dorsal-ventral segregation model in depression (Disner et al, 2011). MDD patients have shown decreased amygdala FC with the dorsal and rostral ACC and DLPFC (Dannlowski et al, 2009; Matthews et al, 2008) and increased amygdala FC with the vmPFC (Almeida et al, 2011; Matthews et al, 2008) during emotional faces processing. Here, in relatives, low negative affect was predicted by high amygdala FC with pgACC, MFG as well as their interaction, suggesting that the increase of amygdala-pgACC FC might 'compensate' for the decrease in amygdala-MFG FC, which would fit previous observations of increased amygdalavmPFC FC as potential resilience mechanism in imaging genetics studies (Heinz et al, 2005; Schardt et al, 2010). Furthermore, the relative decrease of pgACC FC during the faces condition, which was discussed above as a mechanism of automatic attentional control, did not differ between groups, suggesting that this potentially regulatory mechanism is intact in relatives.

In an imaging genetics study of amygdala FC during a comparable faces matching task, amygdala FC increased with supragenual ACC and decreased with sgACC in 5-HTTLPR risk allele carriers across conditions (Pezawas et al, 2005). While the observation of increased amygdala-supragenual ACC FC is in line with our finding (although the effect in Pezawas et al (2005) was slightly more dorsal than the one we observed in the pgACC), the decrease in amygdalasgACC contradicts our finding. Task-dependent amygdalaprefrontal FC should thus be directly compared between patient, familial, and genetic risk groups as soon as genomewide significant variants are identified, to provide conclusive data for the IP hypothesis.

\section{Altered Amygdala FC in Relatives Associated with Faces Processing}

In analyses of group by condition interactions of amygdala FC, relatives showed a differential pattern of conditiondependent modulations of amygdala FC with visual cortex, thalamus, lateral OFC, and SFG. These regions have been described as essential hubs in visual fear processing pathways, in which visual cortex, thalamus and amygdala comprise a 'low-road' input pathway, while lateral OFC and dorsal PFC belong to different regulatory pathways, in which OFC is putatively involved in automatic and PFC in voluntary regulation (Silverstein and Ingvar, 2015). While amygdala FC with visual regions and thalamus was increased in controls during faces matching, which would be in line with increased visual attention due to emotional salience, this pattern was inversed in relatives (reduced amygdala FC during faces processing), suggesting an attenuation or disruption of this input pathway (Silverstein and Ingvar, 2015). Assuming that negative FC reflects an inverse coactivation in terms of an inhibitory process, the strong modulation in OFC (decrease during faces matching in relatives, no difference in controls) and blunted modulation in SFG (decrease during faces matching in controls, no difference in relatives) in relatives suggests a shift from voluntary to automatic regulation pathways in familial risk. Together, this potentially attenuated input processing and increased automatic regulation in relatives might reflect a 'compensatory overregulation' of negative emotional salience. However, since this has been the first report of taskdependent amygdala FC in familial risk for depression, these interpretations must be confirmed by replications and comparisons with patient samples.

\section{Limitations}

With large sample size and the first investigation of condition-dependent changes in amygdala FC as notable strengths, our study is also limited by some aspects: FC is a correlative measure and conclusions regarding causal effects need to be validated by complementary measures. As we did not find associations between amygdala FC and amygdala response in post hoc analyses, we could not provide conclusive data about the implications of FC for brain response. We collapsed siblings, offspring and parents of MDD patients although their levels of depression risk might 
differ due to different genetic and environmental risk loads and previous research focused on offspring. Although siblings and offspring did not differ in our post hoc comparisons, future studies should consider potential differences more thoroughly. Finally, despite exploratory results of brain-behavior associations, our study design did not allow for differentiating between mechanisms of vulnerability and resilience, which should be a research question in future investigations.

\section{CONCLUSIONS}

Our results suggest that aberrant brain activity during emotional processing does not serve as a biomarker of familial risk or intermediate phenotype of genetic risk for MDD. Instead, a dorso-ventral imbalance of amygdala FC across conditions and altered task-dependent modulations with sensory input regions and the prefrontal cortex in familial risk might indicate a stronger involvement of automatic instead of voluntary pathways during emotional processing. To elucidate the clinical implications of these results and to further disentangle mechanisms of vulnerability and resilience, direct comparisons between genetic risk, patient, and control populations and longitudinal studies are recommended.

\section{FUNDING AND DISCLOSURE}

Prof. Meyer-Lindenberg received consultancy fees from: Astra Zeneca, Elsevier, F. Hoffmann-La Roche, Gerson Lehrman Group, Lundbeck Foundation, Outcome Europe Sárl, Outcome Sciences, Roche Pharma, Servier International, and Thieme Verlag, and lecture fees-including the travel fees-from: Abbott, Astra Zeneca, Aula Médica Congresos, BASF, Grupo Ferrer Internacional, Janssen-Cilag, Lilly Deutschland, LVR Klinikum Düsseldorf, Servier Deutschland, Otsuka Pharmaceuticals. Prof. Walter received a speaker honorarium from Servier. Prof. Meyer-Lindenberg and Prof. Walter declared no potential conflict of interest. All other authors reported no biomedical financial compensation or potential conflicts of interest.

\section{ACKNOWLEDGMENTS}

We thank Bob Vogel and Sören Studer for support at Charité Berlin and Leila Haddad, Oliver Grimm and Dagmar Gass for research assistance at ZI Mannheim. The research reported in this study has been funded by the German Federal Ministry for Education and Research (BMBF) grants NGFNplus MooDS (Systematic Investigation of the Molecular Causes of Major Mood Disorders and Schizophrenia) and the Integrated Network IntegraMent (Integrated Understanding of Causes and Mechanisms in Mental Disorders) under the auspices of the e:Med program (grant numbers O1ZX1314B and O1ZX1314G).

\section{REFERENCES}

Almeida JRC de, Sibille EL, Phillips ML (2011). Abnormal left-sided orbitomedial prefrontal cortical-amygdala connectivity during happy and fear face processing: a potential neural mechanism of female MDD. Front Neuropsychiatr Imaging Stimul 2: 69.

Amico F, Carballedo A, Lisiecka D, Fagan AJ, Boyle G, Frodl T (2012). Functional anomalies in healthy individuals with a first degree family history of major depressive disorder. Biol Mood Anxiety Disord 2: 1.

Bastiaansen JA, Servaas MN, Marsman JBC, Ormel J, Nolte IM, Riese $\mathrm{H}$ et al (2014). Filling the gap relationship between the serotonin-transporter-linked polymorphic region and amygdala activation. Psychol Sci 25: 2058-2066.

Bzdok D, Langner R, Schilbach L, Engemann DA, Laird AR, Fox PT et al (2013). Segregation of the human medial prefrontal cortex in social cognition. Front Hum Neurosci 7: 232.

Chen Y, Li H, Jin Z, Shou T, Yu H (2014). Feedback of the amygdala globally modulates visual response of primary visual cortex in the cat. NeuroImage 84: 775-785.

Costa PT, McCrae RR (1992). Normal personality assessment in clinical practice: The NEO Personality Inventory. Psychol Assess 4: 5-13.

Dannlowski U, Ohrmann P, Konrad C, Domschke K, Bauer J, Kugel $\mathrm{H}$ et al (2009). Reduced amygdala-prefrontal coupling in major depression: association with MAOA genotype and illness severity. Int J Neuropsychopharmacol 12: 11-22.

Derogatis L (1977). SCL-90: Administration, Scoring and Procedure Manual-I for the R (Revised) Version. Johns Hopkins University School of Medicine: Baltimore, MD, USA.

Disner SG, Beevers CG, Haigh EAP, Beck AT (2011). Neural mechanisms of the cognitive model of depression. Nat Rev Neurosci 12: 467-477.

Erk S, Meyer-Lindenberg A, Schmierer P, Mohnke S, Grimm O, Garbusow $M$ et al (2014). Hippocampal and frontolimbic function as intermediate phenotype for psychosis: evidence from healthy relatives and a common risk variant in CACNA1C. Biol Psychiatry 76: 466-475.

Etkin A, Egner T, Kalisch R (2011). Emotional processing in anterior cingulate and medial prefrontal cortex. Trends Cogn Sci 15: 85-93.

Foland-Ross LC, Hardin MG, Gotlib IH. Neurobiological markers of familial risk for depression. In: Cowen PJ, Sharp T, Lau JYF (eds). Behavioral neurobiology of depression and its treatment, current topics in behavioral neurosciences. Springer: Berlin Heidelberg, 2012, pp 181-206.

Fornito A, Bullmore ET (2012). Connectomic intermediate phenotypes for psychiatric disorders. Front Neuropsychiatr Imaging Stimul 3: 32.

Friedel E, Schlagenhauf F, Sterzer P, Park SQ, Bermpohl F, Ströhle A et al (2009). 5-HTT genotype effect on prefrontal-amygdala coupling differs between major depression and controls. Psychopharmacology (Berl) 205: 261-271.

Fusar-Poli P, Placentino A, Carletti F, Landi P, Allen P, Surguladze S et al (2009). Functional atlas of emotional faces processing: a voxelbased meta-analysis of 105 functional magnetic resonance imaging studies. J Psychiatry Neurosci 34: 418-432.

Gottesman II, Gould TD (2003). The endophenotype concept in psychiatry: etymology and strategic intentions. Am J Psychiatry 160: 636-645.

Graham J, Salimi-Khorshidi G, Hagan C, Walsh N, Goodyer I, Lennox B et al (2013). Meta-analytic evidence for neuroimaging models of depression: State or trait? J Affect Disord 151: 423-431.

Hariri AR, Bookheimer SY, Mazziotta JC (2000). Modulating emotional responses: effects of a neocortical network on the limbic system. Neuroreport 11: 43-48.

Hariri AR, Mattay VS, Tessitore A, Kolachana B, Fera F, Goldman D et al (2002). Serotonin transporter genetic variation and the response of the human amygdala. Science 297: 400-403.

Hautzinger M, Bailer M, Worrall H, Keller F (1994). BeckDepressions-InveBeck-Depressions-Inventar (BDI). Bearbeitung der Deutschen Ausgabe. Testhandbuch. Huber: Bern, Göttingen, Toronto, Seattle. 
Heinz A, Braus DF, Smolka MN, Wrase J, Puls I, Hermann D et al (2005). Amygdala-prefrontal coupling depends on a genetic variation of the serotonin transporter. Nat Neurosci 8: 20-21.

Holmes TH, Rahe RH (1967). The Social Readjustment Rating Scale. J Psychosom Res 11: 213-218.

Joormann J, Cooney RE, Henry ML, Gotlib IH (2012). Neural correlates of automatic mood regulation in girls at high risk for depression. J Abnorm Psychol 121: 61-72.

Kaiser RH, Andrews-Hanna JR, Wager TD, Pizzagalli DA (2015). Large-scale network dysfunction in major depressive disorder: A meta-analysis of resting-state functional connectivity. JAMA Psychiatry 72: 603-611.

Klein DN, Glenn CR, Kosty DB, Seeley JR, Rohde P, Lewinsohn PM (2013). Predictors of first lifetime onset of major depressive disorder in young adulthood. J Abnorm Psychol 122: 1-6.

Levinson DF, Mostafavi S, Milaneschi Y, Rivera M, Ripke S, Wray NR et al (2014). Genetic studies of major depressive disorder: why are there no genome-wide association study findings and what can we do about it? Biol Psychiatry 76: 510-512.

Li Xa, Sundquist Ka, Hemminki K, Sundquist J (2008). Familial risks for depression among siblings based on hospitalizations in Sweden. Psychiatr Genet 18: 80-84.

Lisiecka DM, Carballedo A, Fagan AJ, Connolly G, Meaney J, Frodl T (2012). Altered inhibition of negative emotions in subjects at family risk of major depressive disorder. J Psychiatr Res 46: 181-188.

Lisiecka DM, Carballedo A, Fagan AJ, Ferguson Y, Meaney J, Frodl $\mathrm{T}$ (2013). Recruitment of the left hemispheric emotional attention neural network in risk for and protection from depression. J Psychiatry Neurosci 38: 117-128.

Mannie ZN, Norbury R, Murphy SE, Inkster B, Harmer CJ, Cowen PJ (2008). Affective modulation of anterior cingulate cortex in young people at increased familial risk of depression. $\mathrm{Br} J$ Psychiatry 192: 356-361.

Mannie ZN, Taylor MJ, Harmer CJ, Cowen PJ, Norbury R (2011). Frontolimbic responses to emotional faces in young people at familial risk of depression. J Affect Disord 130: 127-132.

Mathews A, MacLeod C (2005). Cognitive vulnerability to emotional disorders. Annu Rev Clin Psychol 1: 167-195.

Matthews SC, Strigo IA, Simmons AN, Yang TT, Paulus MP (2008). Decreased functional coupling of the amygdala and supragenual cingulate is related to increased depression in unmedicated individuals with current major depressive disorder. J Affect Disord 111: 13-20.

Mayberg HS (1997). Limbic-cortical dysregulation: a proposed model of depression. J Neuropsychiatry Clin Neurosci 9: 471-481.

McGarry LM, Carter AG (2016). Inhibitory gating of basolateral amygdala inputs to the prefrontal cortex. J Neurosci 36: 9391-9406.

McLaren DG, Ries ML, Xu G, Johnson SC (2012). A generalized form of context-dependent psychophysiological interactions (gPPI): A comparison to standard approaches. NeuroImage 61: 1277-1286.

Meyer-Lindenberg A, Weinberger DR (2006). Intermediate phenotypes and genetic mechanisms of psychiatric disorders. Nat Rev Neurosci 7: 818-827.
Monk PD, Klein PD, Telzer BA, Schroth BA, Mannuzza PD, Moulton I et al (2008). Amygdala and nucleus accumbens activation to emotional facial expressions in children and adolescents at risk for major depression. Am J Psychiatry 165: 90-98.

Petro LS, Smith FW, Schyns PG, Muckli L (2013). Decoding face categories in diagnostic subregions of primary visual cortex. Eur J Neurosci 37: 1130-1139.

Pezawas L, Meyer-Lindenberg A, Drabant EM, Verchinski BA, Munoz KE, Kolachana BS et al (2005). 5-HTTLPR polymorphism impacts human cingulate-amygdala interactions: a genetic susceptibility mechanism for depression. Nat Neurosci 8: 828-834.

Phillips ML, Ladouceur CD, Drevets WC (2008). A neural model of voluntary and automatic emotion regulation: implications for understanding the pathophysiology and neurodevelopment of bipolar disorder. Mol Psychiatry 13: 833-857.

Pilhatsch M, Vetter NC, Hübner T, Ripke S, Müller KU, Marxen M et al (2014). Amygdala-Function Perturbations in Healthy MidAdolescents With Familial Liability for Depression. J Am Acad Child Adolesc Psychiatry 53: 559-568 e6.

Sabatinelli D, Fortune EE, Li Q, Siddiqui A, Krafft C, Oliver WT et al (2011). Emotional perception: Meta-analyses of face and natural scene processing. NeuroImage 54: 2524-2533.

Schardt DM, Erk S, Nüsser C, Nöthen MM, Cichon S, Rietschel M et al (2010). Volition diminishes genetically mediated amygdala hyperreactivity. NeuroImage 53: 943-951.

Silverstein DN, Ingvar M (2015). A multi-pathway hypothesis for human visual fear signaling. Front Syst Neurosci 9: 1-20.

Spielberger CD, Gorsuch RL, Lushene RE (1970). Manual for the State-Trait Anxiety Inventory. Consulting Psychologists Press: Palo Alto, CA, USA.

Stein JL, Wiedholz LM, Bassett DS, Weinberger DR, Zink CF, Mattay VS et al (2007). A validated network of effective amygdala connectivity. NeuroImage 36: 736-745.

Sullivan PF, Daly MJ, O’Donovan M (2012). Genetic architectures of psychiatric disorders: the emerging picture and its implications. Nat Rev Genet 13: 537-551.

Sullivan PF, Neale MC, Kendler KS (2000). Genetic epidemiology of major depression: review and meta-analysis. Am J Psychiatry 157: $1552-1562$.

Whalen PJ, Rauch SL, Etcoff NL, McInerney SC, Lee MB, Jenike MA (1998). Masked presentations of emotional facial expressions modulate amygdala activity without explicit knowledge. J Neurosci 18: 411-418.

Wilde A, Chan H-N, Rahman B, Meiser B, Mitchell PB, Schofield PR et al (2014). A meta-analysis of the risk of major affective disorder in relatives of individuals affected by major depressive disorder or bipolar disorder. J Affect Disord 158: 37-47.

Wittchen H-U, Wunderlich U, Gruschwitz S, Zaudig M (1997). SKID I. Strukturiertes Klinisches Interview für DSM-IV. Achse I: Psychische Störungen. Interviewheft und Beurteilungsheft. Eine deutschsprachige, erweiterte Bearb. d. amerikanischen Original version des SKID I. Hogrefe: Göttingen.

Supplementary Information accompanies the paper on the Neuropsychopharmacology website (http://www.nature.com/npp) 